\title{
Circulating PD-L1 levels change during bevacizumab-based treatment in recurrent glioma
}

\author{
Maximilian J. Mair ${ }^{1,6}$ - Ayseguel Ilhan-Mutlu' ${ }^{1} \cdot$ Sahra Pajenda $^{2} \cdot$ Barbara Kiesel $^{3} \cdot$ Adelheid Wöhrer $^{4} \cdot$ \\ Georg Widhalm $^{3} \cdot$ Karin Dieckmann $^{5} \cdot$ Christine Marosi $^{1} \cdot$ Ludwig Wagner $^{2} \cdot$ Matthias Preusser $^{1,6} \odot$. \\ Anna S. Berghoff ${ }^{1,6}$ (D)
}

Received: 21 December 2020 / Accepted: 26 April 2021 / Published online: 6 May 2021

(c) The Author(s) 2021

\begin{abstract}
Purpose In primary brain tumors, the efficacy of immune-modulating therapies is still under investigation as inflammatory responses are restricted by tight immunoregulatory mechanisms in the central nervous system. Here, we measured soluble PD-L1 (sPD-L1) in the plasma of patients with recurrent glioblastoma (GBM) and recurrent WHO grade II-III glioma treated with bevacizumab-based salvage therapy.

Methods Thirty patients with recurrent GBM and 10 patients with recurrent WHO grade II-III glioma were treated with bevacizumab-based salvage therapy at the Medical University of Vienna. Prior to each treatment cycle, EDTA plasma was drawn and SPD-L1 was measured applying a sandwich ELISA with a lower detection limit of $0.050 \mathrm{ng} / \mathrm{ml}$. Leukocyte counts and C-reactive protein (CRP) levels were measured according to institutional practice.

Results Median number of sPD-L1 measurements was 6 per patient (range: 2-24). At baseline, no significant difference in SPD-L1 concentrations was observed between WHO grade II-III glioma and GBM. Intra-patient variability of sPD-L1 concentrations was significantly higher in WHO grade II-III glioma than in GBM $(p=0.014)$ and tendentially higher in IDH-mutant than in IDH-wildtype glioma ( $p=0.149)$ In WHO grade II-III glioma, sPD-L1 levels were significantly lower after one administration of bevacizumab than at baseline (median: $0.039 \mathrm{ng} / \mathrm{ml} \mathrm{vs.} 0.4855 \mathrm{ng} / \mathrm{ml}, p=0.036$ ). In contrast, no significant change could be observed in patients with GBM.

Conclusions Changes in systemic inflammation markers including sPD-L1 are observable in patients with recurrent glioma under bevacizumab-based treatment and differ between WHO grade II-III glioma and GBM.
\end{abstract}

Keywords Glioma $\cdot$ Soluble PD-L1 $\cdot$ Liquid biomarker $\cdot$ Longitudinal measurement $\cdot$ Systemic inflammation

Anna S. Berghoff

anna.berghoff@meduniwien.ac.at

1 Department of Medicine I, Division of Oncology, Medical University of Vienna, Vienna, Austria

2 Department of Medicine III, Medical University of Vienna, Vienna, Austria

3 Department of Neurosurgery, Medical University of Vienna, Vienna, Austria

4 Department of Neurology, Division of Neuropathology and Neurochemistry, Medical University of Vienna, Vienna, Austria

5 Department of Radiation Oncology, Medical University of Vienna, Vienna, Austria

$6 \quad$ Christian Doppler Laboratory for Personalized Immunotherapy, Medical University of Vienna, Vienna, Austria

$\begin{array}{ll}\text { Abbreviations } \\ \text { 2-HG } & \text { 2-Hydroxyglutarate } \\ \text { CV } & \text { Coefficient of variation } \\ \text { CNS } & \text { Central nervous system } \\ \text { CRP } & \text { C-reactive protein } \\ \text { ELISA } & \text { Enzyme-linked immunosorbent assay } \\ \text { GBM } & \text { Glioblastoma multiforme } \\ \text { IDH } & \text { Isocitrate dehydrogenase } \\ \text { LGG } & \text { Lower-grade glioma (WHO grade II-III glioma) } \\ \text { OS } & \text { Overall survival } \\ \text { PD-L1 } & \text { Programmed death receptor ligand 1 } \\ \text { sPD-L1 } & \text { Soluble PD-L1 } \\ \text { VEGF } & \text { Vascular endothelial growth factor } \\ \text { WHO } & \text { World Health Organization }\end{array}$

Abbreviations

CV Coefficient of variation

CNS Central nervous system

CRP C-reactive protein

ELISA Enzyme-linked immunosorbent assay

GBM Glioblastoma multiforme

IDH Isocitrate dehydrogenase

LGG Lower-grade glioma (WHO grade II-III glioma)

OS Overall survival

PD-L1 Programmed death receptor ligand 1

sPD-L1 Soluble PD-L1

WHO World Health Organization 


\section{Introduction}

Despite optimal surgical and adjuvant treatment, diffuse gliomas have a recurrence rate of up to $90 \%$ due to their infiltrative growth pattern [2]. However, the range of available treatment options at recurrence is very limited, underlining the urgent need for new therapeutic approaches. While immune-modulating therapies have revolutionized oncology, a clinically relevant efficacy in primary brain tumors such as glioma has not been observed so far. The CheckMate-143 trial comparing nivolumab with bevacizumab in recurrent glioma was unable to show a superiority of immune checkpoint inhibition [3]. However, a small subset of patients (objective response rate 7.8\%) showed durable responses, underscoring the need for a more profound understanding of inflammatory subgroups in glioma potentially profiting from immune-modulating therapies [4].

Soluble programmed death receptor ligand 1 (sPD-L1) has been shown to correlate with prognosis and the response toward immune-modulating agents in a wide array of solid tumors [5-9]. Most probably, SPD-L1 is generated by proteolytic cleavage of membrane-bound PD-L1 on both tumor and immune cells. Cleavage may be generated by metalloproteinases such as ADAM10 or ADAM17 [10] whose expression has been described to correlate with poor prognosis in glioma [11]. Previously, we showed that SPD-L1 levels and detectability differ in different brain tumor entities and observed a correlation with survival in lower-grade glioma (LGG) and glioblastoma (GBM) [12]. Specifically, patients with GBM had longer overall survival (OS) in the presence of sPD-1 as compared to their counterparts (median OS 20.9 vs. 8.4 months, $p=0.006$ ). In contrast, patients with LGG presented with worse OS when SPD-L1 could be detected (median OS 38.9 vs. 89.6 months, $p=0.028$ ). These results suggest that the immune phenotype of LGG and GBM might differ, probably due to the immunosuppressive role of the metabolite 2-hydroxyglutarate (2-HG) in isocitrate dehydrogenase (IDH)-mutated tumors [13, 14]. Bevacizumab treatment is frequently used as a salvage treatment in patients with symptomatic glioma progression in need for steroid treatment [15]. Importantly, vascular endothelial growth factor signaling was previously shown to impact the efficacy of immune-modulating therapies in extracranial malignancies [16, 17]. Therefore, we aimed in the present study to investigate the longitudinal SPD-L1 concentrations as a systemic inflammatory marker in patients with recurrent LGG and GBM treated with bevacizumab-based therapy.

\section{Materials and methods}

\section{Patient cohort}

Patients $\geq 18$ years with a recurrence of a histologically confirmed WHO grade II-IV glioma (as determined at the time of first surgery) planned for bevacizumab-based salvage treatment with bevacizumab (either $400 \mathrm{mg}$ or $10 \mathrm{mg}$ / $\mathrm{kg}$ body weight) every 2 weeks were included in this study. Blood samples were drawn before starting systemic bevacizumab-based salvage treatment and prior to each treatment cycle. Other inflammatory markers such as leukocyte counts and C-reactive protein (CRP) levels were concurrently measured at the Department of Laboratory Medicine of the Medical University of Vienna according to institutional practice. All patients were followed up until death. Patient data were stored in a password-secured database (FileMaker Pro Advanced/Server ${ }^{\circledR}$ 17, FileMaker Inc., Santa Clara, CA, USA) and were handled anonymously. The study was conducted according to the Declaration of Helsinki and its amendments as well as to local and institutional guidelines. The study was approved by the ethics committee of the Medical University of Vienna (approval no. 1315/2015).

\section{Immunohistochemistry}

Anti-IDH1 R132H antibody (clone H09, Dianova GmbH, Hamburg, Germany) was used on a Ventana Benchmark Ultra immunostaining platform for evaluation of IDH mutation status [18]. Samples with specific staining in the tumoral region were classified as IDH-mutant (IDH-mt), while negative samples were classified as IDH-wildtype (IDH-wt).

\section{SPD-L1 ELISA}

SPD-L1 levels were measured using a sandwich enzymelinked immunosorbent assay (ELISA) as described previously [12]. Based on the standard dilution curve, a lower limit of sPD-L1 detection of $0.05 \mathrm{ng} / \mathrm{ml}$ was measured.

\section{Statistical analysis}

The coefficients of variation (CV) were calculated to describe the variation of SPD-L1 concentrations within patients over time. Continuous variables were compared between groups by the Mann-Whitney $\mathrm{U}$ and Wilcoxon signed-rank test for unpaired and paired values, respectively. Overall survival (OS) was defined as the time between blood draw for sPD-L1 measurement and allcause death and was compared applying the log-rank test. Results were considered significant at a $p$ value of $\leq 0.05$. As the study was aimed at the generation of hypotheses, no adjustment for multiple testing was performed [19].

Statistical analysis was performed using R 3.6.1 (The R Foundation for Statistical Computing, Vienna, Austria) with RStudio 1.2.1335 (RStudio Inc., Boston, MA, USA) 
and the packages "haven" (version 2.1.1), "ggplot2" (version 3.2.0), "gridExtra" (version 2.3), "GGally" (version 1.4.0), "labelled" (version 2.2.1), "scales" (version 1.0.0), and "survival" (version 2.44-1.1).

\section{Results}

\section{Patients' characteristics}

Thirty patients with recurrent GBM and 10 patients with recurrent WHO grade II-III glioma were included in this study. The baseline characteristics of our cohort are listed in Table 1. Of note, IDH mutational status was available in $35 / 40(87.5 \%)$ patients with $29 / 35(82.9 \%)$ patients with IDH-wt and 6/35 (17.1\%) with IDH-mt glioma. Baseline characteristics according to the presence/absence of IDH mutations are shown in Supplementary Table 1.

\section{sPD-L1 concentrations}

In patients with GBM, the median number of sPD-L1 measurements per patient was 6 (range: $2-16$ ), while in median 5 measurements (range: 2-24) were available in patients with WHO grade II-III glioma. Figure 1 shows a timeline which illustrates survival and the available measurements from the time of treatment initiation.

At baseline, sPD-L1 could be detected in 19/30 (63.3\%) patients with GBM, whereas SPD-L1 could be found in the plasma of $8 / 10(80.0 \%)$ patients with WHO grade II-III glioma ( $p=0.451$, Fisher's exact test). The median sPDL1 concentration in SPD-L1-positive samples at baseline was $0.321 \mathrm{ng} / \mathrm{ml}$ (range: $0.080-42.110 \mathrm{ng} / \mathrm{ml}$ ) in patients

Table 1 Baseline characteristics

\begin{tabular}{|c|c|c|}
\hline & Glioblastoma $(n=30)$ & Lower-grade glioma $(n=10)$ \\
\hline \multicolumn{3}{|l|}{ Gender } \\
\hline Male & $24(80.0 \%)$ & $8(80.0 \%)$ \\
\hline Female & $6(20.0 \%)$ & $2(20.0 \%)$ \\
\hline \multicolumn{3}{|l|}{ Age at first diagnosis (years) } \\
\hline Median (range) & $52(20-75)$ & $47.5(27-56)$ \\
\hline \multicolumn{3}{|l|}{ IDH mutation } \\
\hline IDH-wt & $24 / 30(73.3 \%)$ & $5 / 10(50.0 \%)$ \\
\hline IDH-mt & $1 / 30(3.3 \%)$ & $5 / 10(50.0 \%)$ \\
\hline Unknown & $5 / 30(23.3 \%)$ & $0 / 10(0.0 \%)$ \\
\hline \multicolumn{3}{|l|}{ MGMT promoter methylation status } \\
\hline Methylated & $4(13.3 \%)$ & - \\
\hline Unmethylated & $8(26.7 \%)$ & - \\
\hline Unknown & $18(60.0 \%)$ & $10(100.0 \%)$ \\
\hline \multicolumn{3}{|l|}{ Treatment } \\
\hline Bevacizumab alone & $14(46.7 \%)$ & $5(50.0 \%)$ \\
\hline Bevacizumab + alkylating agent & $9(30.0 \%)$ & $4(40.0 \%)$ \\
\hline Bevacizumab + tyrosine kinase inhibitor & $7(23.3 \%)$ & $1(10.0 \%)$ \\
\hline \multicolumn{3}{|l|}{ Bevacizumab dosage } \\
\hline $400 \mathrm{mg}$ absolute & $16(53.3 \%)$ & $8(80.0 \%)$ \\
\hline$\rightarrow$ Corresponding to mg bevacizumab per kg body weight; median, range & $5.1(4-8.2)$ & $4.7(4-6.7)$ \\
\hline $10 \mathrm{mg} / \mathrm{kg}$ body weight & $14(46.7 \%)$ & $2(20.0 \%)$ \\
\hline \multicolumn{3}{|l|}{ Documented dexamethasone use during study } \\
\hline Yes & $18(60.0 \%)$ & $3(30.0 \%)$ \\
\hline No & $12(40.0 \%)$ & $7(70.0 \%)$ \\
\hline \multicolumn{3}{|l|}{$s P D-L 1$} \\
\hline Detection rates at baseline & $19(63.3 \%)$ & $8(80.0 \%)$ \\
\hline Median (range) of positive samples at baseline [ng/ml] & $0.321(0.080-42.110)$ & $0.658(0.060-2.250)$ \\
\hline Median number of measurements per patient & $6(2-16)$ & $5(2-24)$ \\
\hline Median time to bevacizumab treatment in months (range) & $12.1(5.8-88.6)$ & $37.5(12.1-81.0)$ \\
\hline Median overall survival from first diagnosis (months) & 21.5 (95\% CI: $17.9-31.9)$ & 53.2 (95\% CI: $32.2-72.4)$ \\
\hline Median overall survival from first bevacizumab treatment (months) & 5.3 (95\% CI: 4.7-7.9) & 6.8 (95\% CI: 3.3-14.6) \\
\hline
\end{tabular}




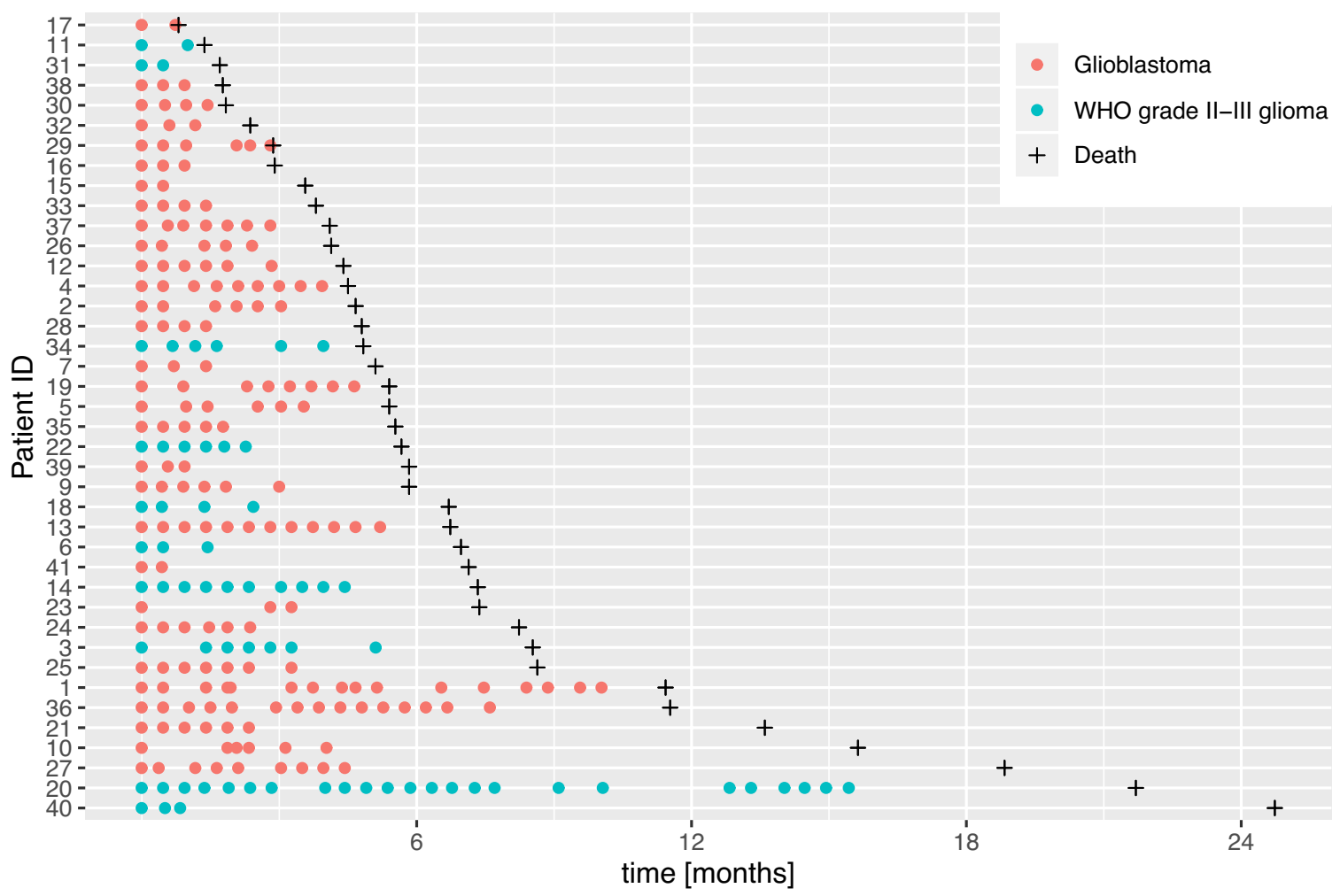

Fig. 1 Available measurements over time $(\bullet)$ and overall survival from first bevacizumab treatment $(+)$ in the study cohort

with GBM and $0.658 \mathrm{ng} / \mathrm{ml}$ (range: $0.060-2.250 \mathrm{ng} / \mathrm{ml}$ ) in patients with WHO grade II-III glioma $(p=0.465$, Mann-Whitney U test; Supplementary Fig. 1A).

In 18/29 (62.1\%) of IDH-wildtype (IDH-wt) patients, sPD-L1 could be detected at baseline while this was the case in $5 / 6(83.3 \%)$ IDH-mutated (IDH-mt) patients $(p=0.64$, Fisher's exact test). Median sPD-L1 concentration in sPD-L1-positive patients at baseline was $0.2795 \mathrm{ng} /$ $\mathrm{ml}$ (range: $0.057-3.383 \mathrm{ng} / \mathrm{ml}$ ) in IDH-wt and $0.563 \mathrm{ng} /$ $\mathrm{ml}$ (range: $0.127-2.245 \mathrm{ng} / \mathrm{ml}$ ) in patients with IDH-mt tumors ( $p=0.551$, Mann-Whitney U test; Supplementary Fig. 1B).

\section{Longitudinal changes in SPD-L1 concentrations over time}

Median CV was 0.63 (range: 0-2.606) in GBM and 1.766 (range: 0.252-3.958) in WHO grade II-III glioma. Intrapatient variation of sPD-L1 concentrations was significantly higher in patients with WHO grade II-III glioma compared to patients with GBM ( $p=0.014$, Mann-Whitney U test; Fig. 2). Moreover, a trend toward higher sPD-L1 variation in IDH-mt (median: 1.71, range: 0.25-3.96) glioma was observed as compared to IDH-wt tumors (median: 0.77, range: $0-3.162 ; p=0.149$ ). No difference in intra-patient variation of sPD-L1 could be observed between patients who received dexamethasone during their treatment (median: 0.64 , range: $0-2.64$ ) and those who did not (median: 0.93, range: $0-3.96 ; p=0.279$ ). Furthermore, $\mathrm{CV}$ did not differ based on whether patients were treated with bevacizumab alone, bevacizumab + alkylating agent or bevacizumab + tyrosine kinase inhibitor $(p=0.764$, Kruskal-Wallis test). A numerical trend towards higher intra-patient variation of sPD-L1 levels was observed in patients who received bevacizumab $10 \mathrm{mg} / \mathrm{kg}$ body weight as compared to patients where $400 \mathrm{mg}$ bevacizumab were administered $(p=0.103$, Mann-Whitney U test).

In GBM, there was no significant difference between pretreatment levels of SPD-L1 and SPD-L1 concentrations before cycle 2 of bevacizumab-based treatment (median SPD-L1 pre-treatment $=0.13 \mathrm{ng} / \mathrm{ml}$ vs. before cycle $2=0.2025 \mathrm{ng} /$ $\mathrm{ml}, p=0.648$, Wilcoxon signed-rank test; Fig. 3a, left panel). In contrast, SPD-L1 levels significantly decreased in WHO grade II-III glioma (median SPD-L1 pre-treatment $=0.4855$ vs. before cycle $2=0.039 ; p=0.036$; Fig. $3 \mathrm{a}$, right panel). Similarly, leukocyte counts significantly diminished in WHO grade II-III glioma (median leukocyte counts pre-treatment $=7.3 \mathrm{G} / 1$ vs. before cycle $2=5.2 \mathrm{G} / \mathrm{l}, p=0.005$; Fig. $3 \mathrm{~b}$, right panel), while there was no significant change in GBM (median pre-treatment $=8.6 \mathrm{G} / \mathrm{l}$ vs. before cycle $2=7.2 \mathrm{G} / \mathrm{l}$, $p=0.859$; Fig. 3b, left panel). In contrast, CRP levels increased in patients with GBM (median pre-treatment $=0.1 \mathrm{mg} / \mathrm{dl}$ vs. 


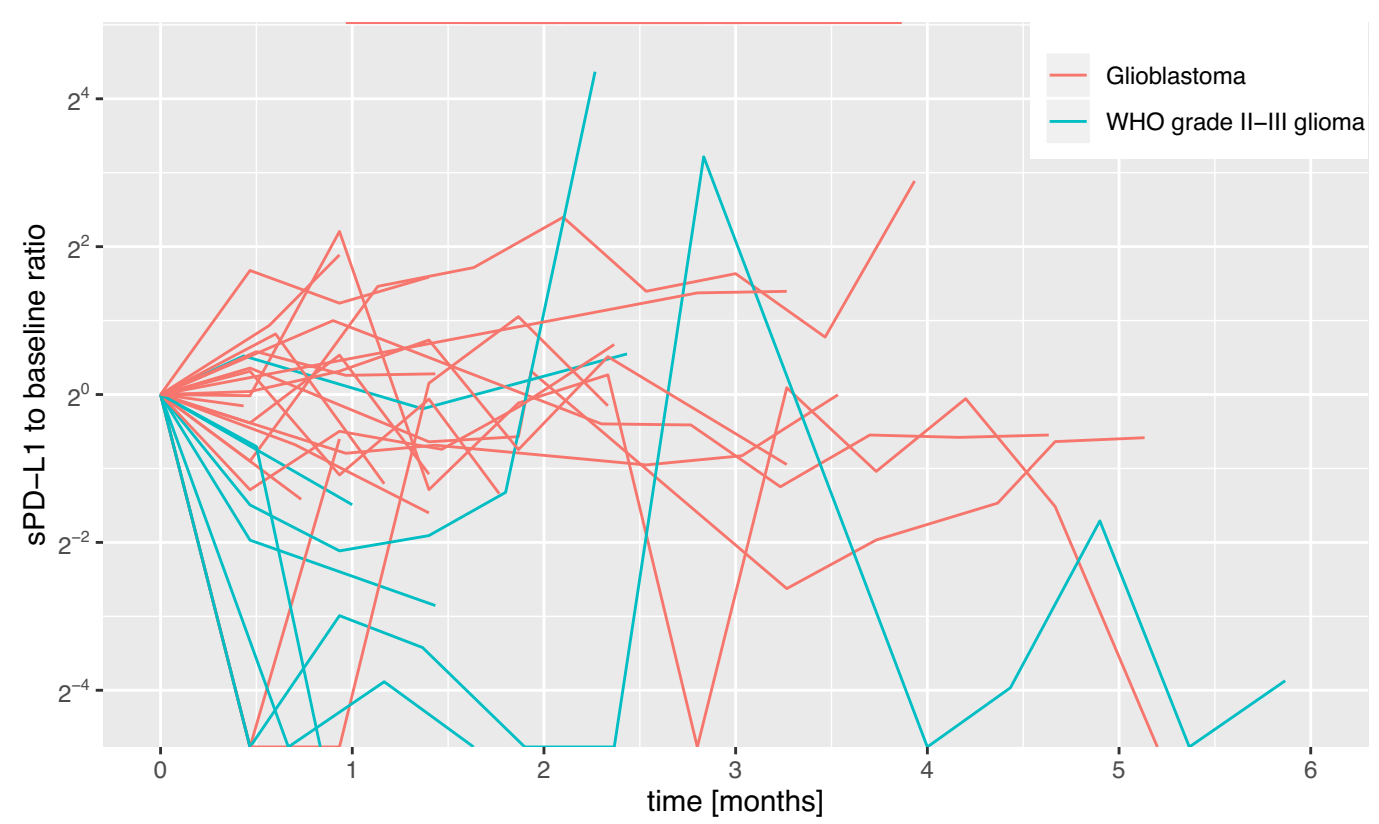

Fig. 2 Spider plot showing the ratios between SPD-L1 concentrations at each timepoint and baseline

A
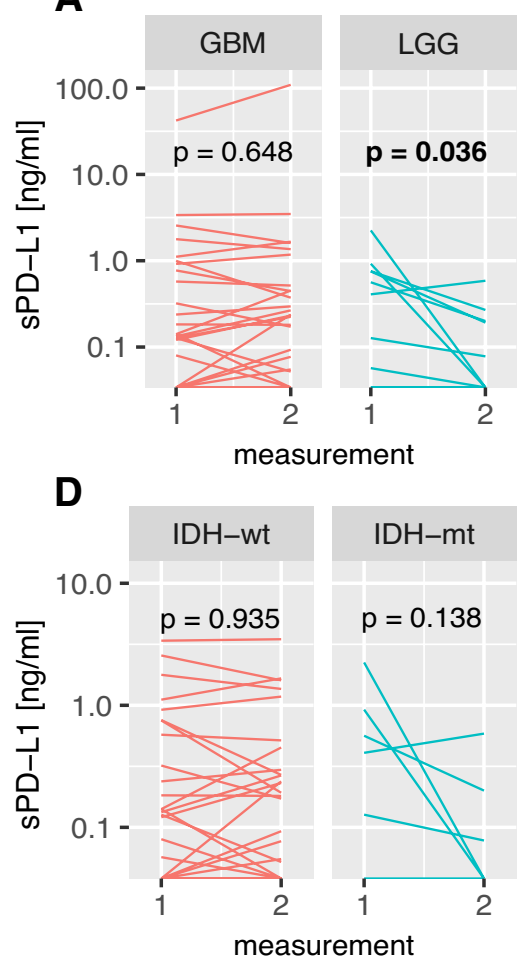

B
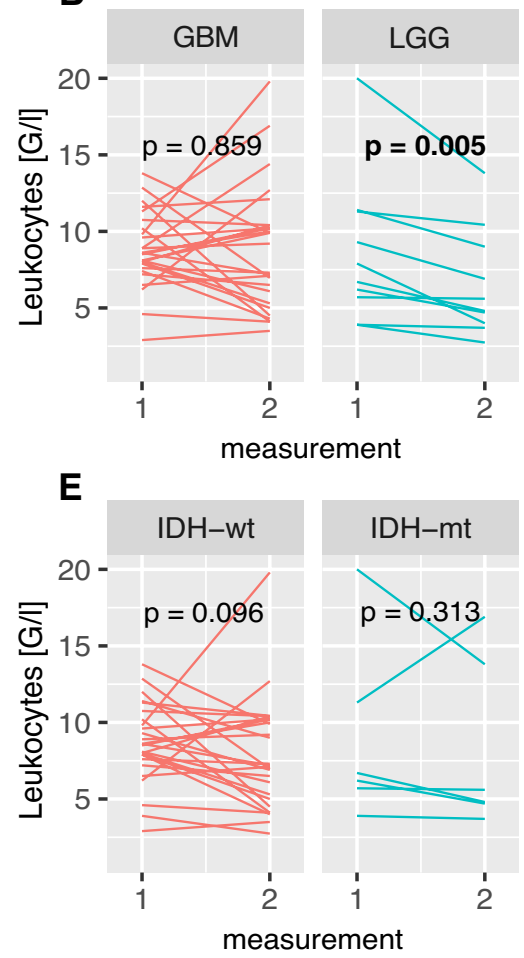
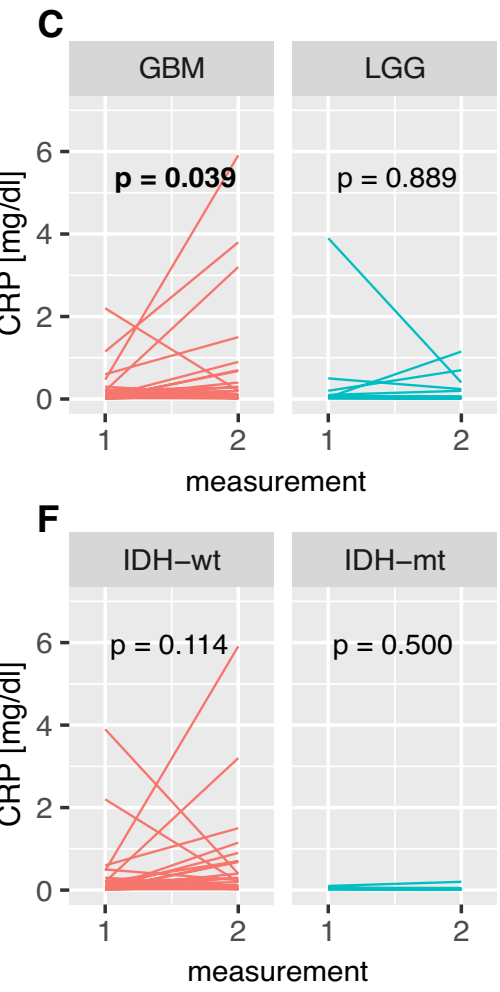

Fig. 3 sPD-L1 concentrations (a/d), leukocyte counts (b/e) and CRP concentrations (c/f) at the first and second measurements in glioblastoma (GBM, a-c, left panel) and WHO grade II-III glioma (lower- grade glioma $=\mathrm{LGG}$, a-c, right panel) as well as IDH-wildtype (IDH-wt, d-f, left panel) and IDH-mutated (IDH-mt, d-f, right panel) glioma. $\mathrm{P}$ values as determined by Wilcoxon signed-rank test 
before cycle $2=0.195 \mathrm{mg} / \mathrm{dl}, p=0.039$; Fig. $3 \mathrm{c}$, left panel), while no significant change could be observed in WHO grade II-III glioma (median pre-treatment $=0.06 \mathrm{mg} / \mathrm{dl}$ vs. before cycle $2=0.13 \mathrm{mg} / \mathrm{dl}, p=0.886$; Fig. $3 \mathrm{c}$, right panel).

No significant differences could be detected between pretreatment and after cycle 1 in both IDH-wt and IDH-mt tumors in terms of sPD-L1, leukocyte counts and CRP levels. However, a numerical trend toward a decrease of sPD-L1 in IDH-mt glioma (median pre-treatment $=0.4855 \mathrm{ng} / \mathrm{ml}$ vs. before cycle $2=0.039 \mathrm{ng} / \mathrm{ml}, p=0.138$; Fig. 3d, right panel) was observed. Furthermore, in IDH-wt tumors, leukocyte counts tendentially decreased (median pre-treatment $=8.6 \mathrm{G} / \mathrm{l}$ vs. before cycle $2=7.03 \mathrm{mg} / \mathrm{dl}, p=0.096$; Fig. 3e, left panel) and CRP levels tendentially increased (median pre-treatment $=0.11 \mathrm{mg} / \mathrm{dl} \mathrm{vs}$. before cycle $2=0.26 \mathrm{mg} / \mathrm{dl}, p=0.114$; Fig. 3f, left panel).

\section{Survival analysis - prognostic impact of SPD-L1}

With respect to overall survival (OS), there were no significant differences between SPD-L1 positive and negative patients at baseline in the GBM ( $p=0.56, \log$-rank test) and WHO grade II-III glioma subgroups $(p=0.68)$ as well as in confirmed IDH-wt cases ( $p=0.63$, Supplementary Fig. 2A-C).

Furthermore, the prognosis of patients with decreasing sPD-L1 in the course of bevacizumab-based therapy did not differ from those where SPD-L1 increased between baseline and the second measurement in both the GBM $(p=0.77)$ and WHO grade II-III sub-cohorts ( $p=0.9$ ) (Supplementary Fig. 2D/E). Similarly, there was no difference in OS in IDHwt glioma according to the change in sPD-L1 levels after the first treatment cycle ( $p=0.65$ ) (Supplementary Fig. 2F). Survival analysis in the IDH-mt subgroup was not performed due to small sample sizes.

\section{Discussion}

In the present study, we analyzed the time course of sPD-L1 as a surrogate marker for systemic inflammation in patients with recurrent glioma receiving bevacizumab-based treatment. As shown previously [12], sPD-L1 is a detectable marker in brain tumors and correlates with local and systemic inflammatory parameters. Here, we found a significant decrease in SPD-L1 levels upon initiation of bevacizumabbased salvage treatment in WHO grade II-III glioma. This change was accompanied by alterations of other established markers of systemic inflammation such as leukocyte counts and CRP levels.

Timely changes of sPD-L1 and other inflammatory parameters in bevacizumab-treated patients with glioma suggest that tumor-immune system interactions are observable on a systemic level. Although gliomas rarely show extracranial metastases and present with a growth pattern restricted to the CNS, several studies previously suggested that systemic inflammation is altered by glioma. Erythrocyte sedimentation rate, CRP levels and the neutrophil-to-lymphocyte ratio were shown to correlate with survival prognosis [20, 21]. Further, an association of systemic inflammation markers such as leukocyte, neutrophil and platelet counts as well as CRP levels with markers of the local tumor microenvironment and overall survival was reported [22]. Indeed, the variability in the systemic inflammation could be an explanation for the so far lacking clinical efficacy of immuno-modulatory therapies in glioma. Systemic inflammation as measured by CRP, neutrophil-to-lymphocyte ratio or recently also SPD-L1 was shown to correlate with the likelihood of response to immune checkpoint inhibitors in extracranial malignancies [23]. Profiling of systemic tumor-immune system interactions could therefore allow for a rational selection of patients who potentially benefit from immunotherapy. Notably, although the CheckMate-143 trial failed to meet its primary endpoint, few durable responses were observed in the nivolumab arm, suggesting that a small subgroup of patients with GBM benefits from immunotherapy [3].

There was higher inter-patient variability of SPD-L1 concentrations in WHO grade II-III glioma than in GBM as determined by the $\mathrm{CV}$. Although not statistically significant, we could detect tendentially higher intra-patient variability in IDH-mt glioma than in IDH-wt tumors. WHO grade II-III gliomas more frequently display IDH mutations leading to an abundant production of the oncometabolite 2-HG exerting local immune-modulating effects. Here, we provide further data on distinct glioma-immune system interactions on systemic level, adding further to the evidence that tumor-immune system interactions differ between glioma subtypes.

In the present study, only patients receiving bevacizumab-based treatment were included. It is well known that therapies targeting the vascular endothelial growth factor (VEGF) axis interact with local and systemic inflammation. Specifically, VEGF inhibition regulates the infiltration of immune cells to the tumor microenvironment by normalizing the tumor vasculature and altering the endothelial expression of cell adhesion molecules [24]. Furthermore, VEGF along with other immunosuppressive factors exerts systemic immunosuppressive effects hampering effective antitumor immune responses [25]. Recently, the results of a phase II trial of pembrolizumab with bevacizumab in recurrent glioma were presented [26]. However, combination therapy was not superior in terms of efficacy as compared to historical bevacizumab monotherapy controls. With regard to our data, it remains elusive whether the observed effects on systemic inflammation are bevacizumab-related or rather due to glioma 
progression, as bevacizumab exerts only minor antitumoral efficacy in glioma and is mainly used for symptomatic control without significant benefit in terms of overall survival [27].

Clearly, our study has several limitations which are mainly due to its exploratory, retrospective design and the small patient number resulting in limited statistical power to detect significant alterations especially in subgroup analyses. The variability in number of measurements per patient with as few as two measurements available in some patients impeded statistical testing for time-dependent effects. Moreover, although we applied the same sandwich ELISA as previously published [12], the method has not been systemically validated with other assays. Lastly, with only 6 IDH-mutant glioma cases, further analyses with respect to molecular subtypes could not be performed.

In conclusion, our data show that tumor-immune system interactions are observable on a systemic level in patients with recurrent glioma. sPD-L1 concentrations change over time in a cohort treated with bevacizumabbased salvage treatment and correlations with other markers of systemic inflammation could be detected, although the mechanistic foundations remain to be elucidated. Our data underscore the need for comprehensive panels of immune-related biomarkers for the conception of prospective immunotherapy trials in CNS tumors. Furthermore, our data add to the available evidence that tumor-immune system interactions differ between glioma subtypes which should be considered in future studies on immune-modulating agents and combinations thereof in glioma.

Supplementary Information The online version contains supplementary material available at https://doi.org/10.1007/s00262-021-02951-2.

Acknowledgements We thank Irene Erber, Stefan Traint, Katharina Feldmann and Teresa Hatziioannou for excellent technical assistance. This study was conducted as a part of the $\mathrm{PhD}$ thesis project "Clinical and immunological characteristics associated with lower-grade glioma prognosis" of Maximilian Mair within the Clinical Neurosciences (CLINS) program at the Medical University of Vienna. Parts of this manuscript were presented as an oral presentation at the European Association of Neuro-Oncology (EANO) Meeting 2019 in Lyon (France) [1].

Author contributions Contribution to study design and its implementation: MJM, AIM, SP, BK, AW, LW, MP, ASB; Data analysis and interpretation: MJM, AIM, SP, BK, AW, GW, KD, CM, LW, MP, ASB; Manuscript writing and editing: MJM, AIM, SP, BK, AW, GW, KD, CM, LW, MP, ASB. All authors read and approved the final version of the manuscript.

Funding Open access funding provided by Medical University of Vienna. The financial support by the Austrian Federal Ministry for Digital and Economic Affairs, the National Foundation for Research, Technology and Development and the Christian Doppler Research Association is gratefully acknowledged.

\section{Declarations}

Conflict of interest Matthias Preusser has received honoraria for lectures, consultation or advisory board participation from the following for-profit companies: Bayer, Bristol-Myers Squibb, Novartis, Gerson Lehrman Group (GLG), CMC Contrast, GlaxoSmithKline, Mundipharma, Roche, BMJ Journals, MedMedia, Astra Zeneca, AbbVie, Lilly, Medahead, Daiichi Sankyo, Sanofi, Merck Sharp \& Dome, Tocagen, Adastra. The following for-profit companies have supported clinical trials and contracted research conducted by MP with payments made to his institution: Boehringer-Ingelheim, Bristol-Myers Squibb, Roche, Daiichi Sankyo, Merck Sharp \& Dome, Novocure, GlaxoSmithKline, AbbVie. Anna Sophie Berghoff has received research support from Daiichi Sankyo and Roche, and honoraria for lectures, consultation or advisory board participation from Roche, Bristol-Myers Squibb, Merck, Daiichi Sankyo as well as travel support from Roche, Amgen and AbbVie. Ayseguel Ilhan-Mutlu participated in advisory boards from Merck Sharp \& Dohme, Bristol-Myers Squibb and Servier, received lecture honoraria from Eli Lilly, Merck Sharp \& Dohme and Servier, is the local PI for clinical trials sponsored by Bristol-Myers Squibb and Roche and received travel support from Bristol-Myers Squibb and Roche. All other authors declare that they have no conflict of interest related to the present study.

Ethical approval This study was performed according to the ethical standards of the Ethics Committee of the Medical University of Vienna (approval no. 1315/2015) and the Helsinki Declaration of 1964 and its amendments.

Open Access This article is licensed under a Creative Commons Attribution 4.0 International License, which permits use, sharing, adaptation, distribution and reproduction in any medium or format, as long as you give appropriate credit to the original author(s) and the source, provide a link to the Creative Commons licence, and indicate if changes were made. The images or other third party material in this article are included in the article's Creative Commons licence, unless indicated otherwise in a credit line to the material. If material is not included in the article's Creative Commons licence and your intended use is not permitted by statutory regulation or exceeds the permitted use, you will need to obtain permission directly from the copyright holder. To view a copy of this licence, visit http://creativecommons.org/licenses/by/4.0/.

\section{References}

1. Mair M, Ilhan-Mutlu A, Pajenda S et al (2019) OS1.1 Plasma PD-L1 levels over time differ between glioblastoma and lowergrade glioma patients. Neuro Oncol. https://doi.org/10.1093/neuonc/noz126.014

2. Minniti G, Amelio D, Amichetti M et al (2010) Patterns of failure and comparison of different target volume delineations in patients with glioblastoma treated with conformal radiotherapy plus concomitant and adjuvant temozolomide. Radiother Oncol 97:377-381. https://doi.org/10.1016/j.radonc.2010.08.020

3. Reardon DA, Brandes AA, Omuro A et al (2020) Effect of nivolumab vs bevacizumab in patients with recurrent glioblastoma: the checkmate 143 phase 3 randomized clinical trial. JAMA Oncol. https://doi.org/10.1001/jamaoncol.2020.1024

4. Reardon DA, Omuro A, Brandes AA et al (2017) OS10.3 randomized phase 3 study evaluating the efficacy and safety of nivolumab vs bevacizumab in patients with recurrent glioblastoma: checkmate 143. Neuro Oncol 19:III21-III21 
5. Finkelmeier F, Canli Ö, Tal A et al (2016) High levels of the soluble programmed death-ligand (sPD-L1) identify hepatocellular carcinoma patients with a poor prognosis. Eur J Cancer 59:152-159. https://doi.org/10.1016/j.ejca.2016.03.002

6. Zhou J, Mahoney KM, Giobbie-Hurder A et al (2017) Soluble PD-L1 as a biomarker in malignant melanoma treated with checkpoint blockade. Cancer Immunol Res 5:480-492. https://doi.org/ 10.1158/2326-6066.cir-16-0329

7. Shigemori T, Toiyama Y, Okugawa Y et al (2019) Soluble PD-L1 expression in circulation as a predictive marker for recurrence and prognosis in gastric cancer: direct comparison of the clinical burden between tissue and serum PD-L1 expression. Ann Surg Oncol 26:876-883. https://doi.org/10.1245/s10434-018-07112-x

8. Fukuda T, Kamai T, Masuda A et al (2016) Higher preoperative serum levels of PD-L1 and B7-H4 are associated with invasive and metastatic potential and predictable for poor response to VEGF-targeted therapy and unfavorable prognosis of renal cell carcinoma. Cancer Med 5:1810-1820. https://doi.org/10.1002/ cam4.754

9. Bian B, Fanale D, Dusetti N et al (2019) Prognostic significance of circulating PD-1, PD-L1, pan-BTN3As, BTN3A1 and BTLA in patients with pancreatic adenocarcinoma. Oncoimmunology. https://doi.org/10.1080/2162402X.2018.1561120

10. Orme JJ, Jazieh KA, Xie T et al (2020) ADAM10 and ADAM17 cleave PD-L1 to mediate PD-(L)1 inhibitor resistance. Oncoimmunology. https://doi.org/10.1080/2162402X.2020.1744980

11. Wu B, Sha L, Wang Y et al (2014) Diagnostic and prognostic value of a disintegrin and metalloproteinase-17 in patients with gliomas. Oncol Lett 8:2616-2620. https://doi.org/10.3892/ol. 2014.2582

12. Mair MJ, Pajenda S, Ilhan-Mutlu A et al (2020) Soluble PD-L1 is associated with local and systemic inflammation markers in primary and secondary brain tumours. ESMO open. https://doi. org/10.1136/esmoopen-2020-000863

13. Bunse L, Pusch S, Bunse T et al (2018) Suppression of antitumor T cell immunity by the oncometabolite (R)-2-hydroxyglutarate. Nat Med 24:1192-1203. https://doi.org/10.1038/s41591-018-0095-6

14. Berghoff AS, Kiesel B, Widhalm G et al (2017) Correlation of immune phenotype with IDH mutation in diffuse glioma. Neuro Oncol 19:1460-1468. https://doi.org/10.1093/neuonc/nox054

15. Weller M, Le Rhun E, Preusser M et al (2019) How we treat glioblastoma. ESMO Open 4:1-4. https://doi.org/10.1136/esmoo pen-2019-000520

16. Socinski MA, Jotte RM, Cappuzzo F et al (2018) Atezolizumab for first-line treatment of metastatic nonsquamous NSCLC. N Engl J Med 378:2288-2301. https://doi.org/10.1056/NEJMoa1716 948
17. Rini BI, Plimack ER, Stus V et al (2019) Pembrolizumab plus axitinib versus sunitinib for advanced renal-cell carcinoma. N Engl J Med 380:1116-1127. https://doi.org/10.1056/NEJMoa1816714

18. Capper D, Weissert S, Balss J et al (2010) Characterization of R132H mutation-specific IDH1 antibody binding in brain tumors. Brain Pathol 20:245-254. https://doi.org/10.1111/j.1750-3639. 2009.00352.x

19. Bender R, Lange S (2001) Adjusting for multiple testing-When and how? J Clin Epidemiol 54:343-349. https://doi.org/10.1016/ S0895-4356(00)00314-0

20. Strojnik T, Šmigoc T, Lah TT (2014) Prognostic value of erythrocyte sedimentation rate and C-reactive protein in the blood of patients with glioma. Anticancer Res 34:339-347

21. Han S, Liu Y, Li Q et al (2015) Pre-treatment neutrophil-to-lymphocyte ratio is associated with neutrophil and T-cell infiltration and predicts clinical outcome in patients with glioblastoma. BMC Cancer 15:1-10. https://doi.org/10.1186/s12885-015-1629-7

22. Mir Seyed Nazari P, Ay C, Preusser M et al (2019) Association of systemic inflammation with local tumour characteristics and survival in glioma patients. Ann Oncol. https://doi.org/10.1093/ annonc/mdz243.009

23. Mazzaschi G, Minari R, Zecca A et al (2020) Soluble PD-L1 and circulating CD8+PD-1+ and NK Cells enclose a prognostic and predictive immune effector score in immunotherapy treated NSCLC patients. Lung Cancer. https://doi.org/10.1016/j.lungcan. 2020.07.028

24. Melder RJ, Koenig GC, Witwer BP et al (1996) During angiogenesis, vascular endothelial growth factor and basic fibroblast growth factor regulate natural killer cell adhesion to tumor endothelium. Nat Med 2:992-997. https://doi.org/10.1038/nm0996-992

25. Fukumura D, Kloepper J, Amoozgar Z et al (2018) Enhancing cancer immunotherapy using antiangiogenics: opportunities and challenges. Nat Rev Clin Oncol 15:325-340. https://doi.org/10. 1038/nrclinonc.2018.29

26. Reardon DA, Nayak L, Peters KB et al (2018) Phase II study of pembrolizumab or pembrolizumab plus bevacizumab for recurrent glioblastoma (rGBM) patients. J Clin Oncol 36:2006. https://doi. org/10.1200/JCO.2018.36.15_suppl.2006

27. Weller M, van den Bent M, Tonn JC et al (2017) European association for neuro-oncology (EANO) guideline on the diagnosis and treatment of adult astrocytic and oligodendroglial gliomas. Lancet Oncol 18:e315-e329. https://doi.org/10.1016/S14702045(17)30194-8

Publisher's Note Springer Nature remains neutral with regard to jurisdictional claims in published maps and institutional affiliations. 